\title{
CORRECTION
}

\section{Correction to: Brain Tumor Detection and Classification Using Cycle Generative Adversarial Networks}

\author{
Rajeev Kumar Gupta ${ }^{1}$ (D) Santosh Bharti ${ }^{1}$. Nilesh Kunhare ${ }^{2} \cdot$ Yatendra Sahu $^{3} \cdot$ Nikhlesh Pathik $^{4}$
}

Published online: 21 February 2022

(C) International Association of Scientists in the Interdisciplinary Areas 2022

\section{Correction to: \\ Interdisciplinary Sciences: Computational Life Sciences \\ https://doi.org/10.1007/s12539-022-00502-6}

In the Original publication, the author has found few errors as below:

1. The 3rd author name has been misspelled as Nilesk Kunhare. The corrected name should appear as Nilesh Kunhare.

2. The affiliation of author 3 and author 4 has been interchanged. The correct name and affiliation of authors 2 and 3 should appear as:

Author 3: Nilesh Kunhare: Amity University, Gwalior

Author 4: Yatendra Sahu: IIIT-Bhopal

The original article has been corrected.

The original article can be found online at https://doi.org/10.1007/ s12539-022-00502-6.

Rajeev Kumar Gupta

Rajeevmanit12276@gmail.com

Santosh Bharti

santosh.bharti@sot.pdpu.ac.in

Nilesh Kunhare

nilesh954@gmail.com

Yatendra Sahu

yatendra.sati@gmail.com

Nikhlesh Pathik

pathiknikhlesh@gmail.com

1 Pandit Deendayal Energy University, Gandhinagar, India

2 Amity University, Gwalior, India

3 IIIT, Bhopal, India

4 Sagar Institute of Science \& Technology, Bhopal, India 